\title{
A Sharp Double Inequality between Seiffert, Arithmetic, and Geometric Means
}

\author{
Wei-Ming Gong, ${ }^{1}$ Ying-Qing Song, ${ }^{1}$ \\ Miao-Kun Wang, ${ }^{2}$ and Yu-Ming Chu ${ }^{2}$ \\ ${ }^{1}$ College of Mathematics and Computation Science, Hunan City University, Yiyang 413000, China \\ ${ }^{2}$ Department of Mathematics, Huzhou Teachers College, Huzhou 313000, China
}

Correspondence should be addressed to Yu-Ming Chu, chuyuming2005@yahoo.com.cn

Received 2 July 2012; Accepted 21 August 2012

Academic Editor: Josef Diblík

Copyright (C) 2012 Wei-Ming Gong et al. This is an open access article distributed under the Creative Commons Attribution License, which permits unrestricted use, distribution, and reproduction in any medium, provided the original work is properly cited.

For fixed $s \geq 1$ and any $t_{1}, t_{2} \in(0,1 / 2)$ we prove that the double inequality $G^{s}\left(t_{1} a+\left(1-t_{1}\right) b, t_{1} b+\right.$ $\left.\left(1-t_{1}\right) a\right) A^{1-s}(a, b)<P(a, b)<G^{s}\left(t_{2} a+\left(1-t_{2}\right) b, t_{2} b+\left(1-t_{2}\right) a\right) A^{1-s}(a, b)$ holds for all $a, b>0$ with $a \neq b$ if and only if $t_{1} \leq\left(1-\sqrt{1-(2 / \pi)^{2 / s}}\right) / 2$ and $t_{2} \geq(1-1 / \sqrt{3 s}) / 2$. Here, $P(a, b), A(a, b)$ and $G(a, b)$ denote the Seiffert, arithmetic, and geometric means of two positive numbers $a$ and $b$, respectively.

\section{Introduction}

The Seiffert mean $P(a, b)$ [1] of two distinct positive numbers $a$ and $b$ is defined by

$$
P(a, b)=\frac{a-b}{4 \arctan (\sqrt{a / b})-\pi}
$$

Recently, the Seiffert mean $P(a, b)$ has been the subject of intensive research. In particular, many remarkable inequalities for $P(a, b)$ can be found in the literature [2-17]. The Seiffert mean $P(a, b)$ can be rewritten as (see $[6,(2.4)]$ )

$$
P(a, b)=\frac{a-b}{2 \arcsin ((a-b) /(a+b))} .
$$


Let $A(a, b)=(a+b) / 2, G(a, b)=\sqrt{a b}$ and $H(a, b)=2 a b /(a+b)$ be the classical arithmetic, geometric, and harmonic means of two positive numbers $a$ and $b$, respectively. Then it is well known that inequalities $H(a, b)<G(a, b)<P(a, b)<A(a, b)$ hold for all $a, b>0$ with $a \neq b$.

For $\alpha, \beta, \lambda, \mu \in(0,1 / 2)$, Chu et al. [18, 19] proved that the double inequalities

$$
\begin{aligned}
& G(\alpha a+(1-\alpha) b, \alpha b+(1-\alpha) a)<P(a, b)<G(\beta a+(1-\beta) b, \beta b+(1-\beta) a) \\
& H(\lambda a+(1-\lambda) b, \lambda b+(1-\lambda) a)<P(a, b)<H(\mu a+(1-\mu) b, \mu b+(1-\mu) a)
\end{aligned}
$$

hold for all $a, b>0$ with $a \neq b$ if and only if $\alpha \leq\left(1-\sqrt{1-4 / \pi^{2}}\right) / 2, \beta \geq(3-\sqrt{3}) / 6, \lambda \leq$ $(1-\sqrt{1-2 / \pi}) / 2$ and $\mu \geq(6-\sqrt{6}) / 12$.

Let $t \in(0,1 / 2), s \geq 1$ and

$$
Q_{t, s}(a, b)=G^{s}(t a+(1-t) b, t b+(1-t) a) A^{1-s}(a, b),
$$

then it is not difficult to verify that

$$
\begin{aligned}
& Q_{t, 1}(a, b)=G(t a+(1-t) b, t b+(1-t) a), \\
& Q_{t, 2}(a, b)=H(t a+(1-t) b, t b+(1-t) a)
\end{aligned}
$$

and $Q_{t, s}(a, b)$ is strictly increasing with respect to $t \in(0,1 / 2)$ for fixed $a, b>0$ with $a \neq b$.

It is natural to ask what are the largest value $t_{1}=t_{1}(s)$ and the least value $t_{2}=t_{2}(s)$ in $(0,1 / 2)$ such that the double inequality $Q_{t_{1}, s}(a, b)<P(a, b)<Q_{t_{2}, s}(a, b)$ holds for all $a, b>0$ with $a \neq b$ and $s \geq 1$. The main purpose of this paper is to answer this question.

\section{Main Result}

In order to establish our main result we need two lemmas, which we present in the following.

Lemma 2.1. If $s \geq 1$, then $1 /(3 s)+(2 / \pi)^{2 / s}<1$.

Proof. Consider the following:

$$
f(s)=\frac{1}{3 s}+\left(\frac{2}{\pi}\right)^{2 / s}
$$


Then simple computations lead to

$$
\begin{aligned}
\lim _{s \rightarrow+\infty} f(s)=1 \\
f^{\prime}(s)=\frac{2}{s^{2}} \log \frac{\pi}{2}\left[\left(\frac{2}{\pi}\right)^{2 / s}-\frac{1}{6 \log (\pi / 2)}\right] \\
\geq \frac{2}{s^{2}} \log \frac{\pi}{2}\left[\left(\frac{2}{\pi}\right)^{2}-\frac{1}{6 \log (\pi / 2)}\right] \\
=\frac{24 \log (\pi / 2)-\pi^{2}}{3 \pi^{2} s^{2}}
\end{aligned}
$$

for $s \geq 1$.

Computational and numerical experiments show that

$$
24 \log \left(\frac{\pi}{2}\right)-\pi^{2}=0.968 \cdots>0
$$

Inequalities (2.3) and (2.4) imply that $f(s)$ is strictly increasing in $[1,+\infty)$. Therefore, Lemma 2.1 follows from (2.1) and (2.2) together with the monotonicity of $f(s)$.

Lemma 2.2. Let $0 \leq u \leq 1, s \geq 1$ and

$$
f_{u, s}(x)=\frac{s}{2} \log \left(1-u x^{2}\right)-\log x+\log (\arcsin x) .
$$

Then inequality $f_{u, s}(x)>0$ holds for all $x \in(0,1)$ if and only if $3 s u \leq 1$, and inequality $f_{u, s}(x)<0$ holds for all $x \in(0,1)$ if and only if $u+(2 / \pi)^{2 / s} \geq 1$.

Proof. If $u=0$, then we clearly see that $3 s u \leq 1, u+(2 / \pi)^{2 / s}<1$ and $f_{0, s}(x)=$ $\log [(\arcsin x) / x]>0$ for all $s \geq 1$ and $x \in(0,1)$. In the following discussion, we assume that $0<u \leq 1$.

From (2.5) and simple computations we have

$$
\begin{gathered}
\lim _{x \rightarrow 0^{+}} f_{u, s}(x)=0, \\
f_{u, s}^{\prime}(x)=\frac{1}{\sqrt{1-x^{2}} \arcsin x}-\frac{1+u(s-1) x^{2}}{x\left(1-u x^{2}\right)}=\frac{1+u(s-1) x^{2}}{x\left(1-u x^{2}\right) \arcsin x} g_{u, s}(x),
\end{gathered}
$$


where

$$
\begin{aligned}
& g_{u, s}(x)=\frac{x\left(1-u x^{2}\right)}{\sqrt{1-x^{2}}\left[1+u(s-1) x^{2}\right]}-\arcsin x, \\
& g_{u, s}(0)=0, \\
& g_{u, s}^{\prime}(x)=\frac{x^{2}}{\left(1-x^{2}\right)^{3 / 2}\left[1+u(s-1) x^{2}\right]^{2}} h_{u, s}(x),
\end{aligned}
$$

where

$$
\begin{gathered}
h_{u, s}(x)=u^{2}(s-1)^{2} x^{4}+u\left(-s^{2} u+u s+4 s-2\right) x^{2}+1-3 s u, \\
h_{u, s}(0)=1-3 s u, \\
h_{u, s}(1)=u s(1-u)+(1-u)^{2} .
\end{gathered}
$$

We divide the proof into four cases.

Case $1(3 s u \leq 1)$. Then from (2.11) and (2.12) together with the fact that

$$
-u s^{2}+u s+4 s-2=2(s-1)+s(u+2 s u+1)+s(1-3 s u)>0,
$$

we clearly see that

$$
h_{u, s}(0) \geq 0,
$$

and $h_{u, s}(x)$ is strictly increasing in $[0,1]$.

Equation (2.12) and the monotonicity of $h_{u, s}(x)$ imply that

$$
h_{u, s}(x)>0
$$

for $x \in(0,1]$.

Equation (2.10) and inequality (2.16) lead to the conclusion that $g_{u, s}(x)$ is strictly increasing in $[0,1)$. Then from $(2.9)$ we know that

$$
g_{u, s}(x)>0
$$

for $x \in(0,1)$.

It follows from (2.7) and inequality $(2.17)$ that $f_{u, s}(x)$ is strictly increasing in $(0,1]$.

Therefore, $f_{u, s}(x)>0$ for all $x \in(0,1)$ follows from (2.6) and the monotonicity of $f_{u, s}(x)$.

Case $2(3 s u>1)$. Then $(2.12)$ and the continuity of $h_{u, s}(x)$ imply that there exists $0<\lambda<1$ such that

$$
h_{u, s}(x)<0
$$

for $x \in[0, \lambda)$. 
Therefore, $f_{u, s}(x)<0$ for $x \in(0, \lambda)$ follows easily from (2.6), (2.7), (2.9) and (2.10) together with inequality (2.18).

Case $3\left(u+(2 / \pi)^{2 / s} \geq 1\right)$. Then Lemma 2.1 and (2.12) lead to

$$
h_{u, s}(0)=1-3 s u \leq 1-3 s\left[1-\left(\frac{2}{\pi}\right)^{2 / s}\right]<0 .
$$

We divide the proof into two subcases.

Subcase $3.1(u=1)$. Then (2.13) becomes

$$
h_{u, s}(1)=0
$$

Let $t=x^{2}$, then from (2.11) we clearly see that the function $h_{u, s}$ is a quadratic function of variable $t$. It follows from inequality (2.19) and (2.20) that

$$
h_{u, s}(x)<0
$$

for all $x \in[0,1)$.

Therefore, $f_{u, s}(x)<0$ for $x \in(0,1)$ follows easily from (2.6), (2.7), (2.9) and (2.10) together with inequality (2.21).

Subcase $3.2(0<u<1)$. Then from (2.5), (2.8), and (2.13) we have

$$
\begin{gathered}
f_{u, s}(1)=\log \left[\frac{\pi}{2}(1-u)^{s / 2}\right] \leq 0, \\
\lim _{x \rightarrow 1^{-}} g_{u, s}(x)=+\infty, \\
h_{u, s}(1)>0 .
\end{gathered}
$$

From (2.11), (2.19), and (2.24) we clearly see that there exists $0<\lambda_{1}<1$ such that $h_{u, s}(x)<0$ for $x \in\left[0, \lambda_{1}\right)$ and $h_{u, s}(x)>0$ for $x \in\left(\lambda_{1}, 1\right]$. Then (2.10) implies that $g_{u, s}(x)$ is strictly decreasing in $\left[0, \lambda_{1}\right]$ and strictly increasing in $\left[\lambda_{1}, 1\right)$.

From (2.9) and (2.23) together with the piecewise monotonicity of $g_{u, s}(x)$ we clearly see that there exists $0<\lambda_{2}<1$ such that $g_{u, s}(x)<0$, for $x \in\left(0, \lambda_{2}\right)$ and $g_{u, s}(x)>0$ for $x \in\left(\lambda_{2}, 1\right)$. Then (2.7) implies that $f_{u, s}(x)$ is strictly decreasing in $\left(0, \lambda_{2}\right]$ and strictly increasing in $\left[\lambda_{2}, 1\right]$.

Therefore, $f_{u, s}(x)<0$ for $x \in(0,1)$ follows from (2.6) and (2.22) together with the piecewise monotonicity of $f_{u, s}(x)$.

Case $4\left(u+(2 / \pi)^{2 / s}<1\right)$. Then $(2.5)$ leads to

$$
f_{u, s}(1)=\log \left[\frac{\pi}{2}(1-u)^{s / 2}\right]>0
$$

From inequality (2.25) and the continuity of $f_{u, s}(x)$ we know that there exists $0<\mu<1$ such that $f_{u, s}(x)>0$ for $x \in(\mu, 1]$. 
Theorem 2.3. If $t_{1}, t_{2} \in(0,1 / 2)$ and $s \geq 1$, then the double inequality

$$
Q_{t_{1, s}}(a, b)<P(a, b)<Q_{t_{2, s}}(a, b)
$$

holds for all $a, b>0$ with $a \neq b$ if and only if $t_{1} \leq\left(1-\sqrt{1-(2 / \pi)^{2 / s}}\right) / 2$ and $t_{2} \geq(1-1 / \sqrt{3 s}) / 2$.

Proof. Since both $Q_{t, s}(a, b)$ and $P(a, b)$ are symmetric and homogeneous of degree 1 . Without loss of generality, we assume that $a>b$. Let $x=(a-b) /(a+b) \in(0,1)$. Then from (1.2) and (1.4) we have

$$
\begin{aligned}
\log \left(\frac{Q_{t, S}(a, b)}{P(a, b)}\right) & =\log \left(\frac{Q_{t, s}(a, b)}{A(a, b)}\right)-\log \left(\frac{P(a, b)}{A(a, b)}\right) \\
& =\frac{S}{2} \log \left[1-(1-2 t)^{2} x^{2}\right]-\log x+\log (\arcsin x) .
\end{aligned}
$$

Therefore, Theorem 2.3 follows easily from Lemma 2.2 and (2.27).

\section{Acknowledgments}

This research was supported by the Natural Science Foundation of China under Grant 11071069 and the Natural Science Foundation of Hunan Province under Grant 09JJ6003.

\section{References}

[1] H. J. Seiert, "Problem 887," Nieuw Archief Voor Wiskunde (4), vol. 11, no. 2, article 176, 1993.

[2] H. J. Seiert, “Aufgabe $\beta 16, "$ Die Wurzel, vol. 29, pp. 221-222, 1995.

[3] G. Toader, "Seiffert type means," Nieuw Archief voor Wiskunde (4), vol. 17, no. 3, pp. 379-382, 1999.

[4] P. A. Hästö, "A monotonicity property of ratios of symmetric homogeneous means," Journal of Inequalities in Pure and Applied Mathematics, vol. 3, no. 5, article 71, 23 pages, 2002.

[5] E. Neuman and J. Sándor, "On certain means of two arguments and their extensions," International Journal of Mathematics and Mathematical Sciences, no. 16, pp. 981-993, 2003.

[6] E. Neuman and J. Sándor, “On the Schwab-Borchardt mean," Mathematica Pannonica, vol. 14, no. 2, pp. 253-266, 2003.

[7] P. A. Hästö, "Optimal inequalities between Seiffert's mean and power means," Mathematical Inequalities \& Applications, vol. 7, no. 1, pp. 47-53, 2004.

[8] Y. M. Chu, Y. F. Qiu, and M. K. Wang, "Sharp power mean bounds for the combination of Seiffert and geometric means," Abstract and Applied Analysis, vol. 2010, Article ID 108920, 12 pages, 2010.

[9] Y. M. Chu, Y. F. Qiu, M. K. Wang, and G. D. Wang, "The optimal convex combination bounds of arithmetic and harmonic means for the Seiffert's mean," Journal of Inequalities and Applications, vol. 2010, Article ID 436457, 7 pages, 2010.

[10] S. S. Wang and Y. M. Chu, "The best bounds of the combination of arithmetic and harmonic means for the Seiffert's mean," International Journal of Mathematical Analysis, vol. 4, no. 21-24, pp. 1079-1084, 2010.

[11] C. Zong and Y. M. Chu, "An inequality among identric, geometric and Seiffert's means," International Mathematical Forum, vol. 5, no. 25-28, pp. 1297-1302, 2010.

[12] M. K. Wang, Y. F. Qiu, and Y. M. Chu, "Sharp bounds for Seiffert means in terms of Lehmer means," Journal of Mathematical Inequalities, vol. 4, no. 4, pp. 581-586, 2010.

[13] H. Liu and X. J. Meng, "The optimal convex combination bounds for Seiffert's mean," Journal of Inequalities and Applications, vol. 2011, Article ID 686834, 9 pages, 2011.

[14] Y. M. Chu, M. K. Wang, and W. M. Gong, "Two sharp double inequalities for Seiffert mean," Journal of Inequalities and Applications, vol. 2011, article 44, 7 pages, 2011. 
[15] H. N. Hu, S. W. Hou, Y. W. Xu, and Y. M. Chu, “Optimal convex combination bounds of root-square and harmonic root-square means for Seiffert mean," International Mathematical Forum, vol. 6, no. 5760, pp. 2823-2831, 2011.

[16] Y. F. Qiu, M. K. Wang, and Y. M. Chu, "The sharp combination bounds of arithmetic and logarithmic means for Seiffert's mean," International Journal of Pure and Applied Mathematics, vol. 72, no. 1, pp. 11-18, 2011.

[17] S. Q. Gao, "Inequalities for the Seiffert's means in terms of the identric mean," Journal of Mathematical Sciences, Advances and Applications, vol. 10, no. 1-2, pp. 23-31, 2011.

[18] Y. M. Chu, M. K. Wang, and Z. K. Wang, "An optimal double inequality between Seiffert and geometric means," Journal of Applied Mathematics, vol. 2011, Article ID 261237, 6 pages, 2011.

[19] Y. M. Chu, M. K. Wang, and Z. K. Wang, "A best-possilbe double inequality between Seiffert and harmonic means," Journal of Inequalities and Applications, vol. 2011, article 94, 7 pages, 2011. 


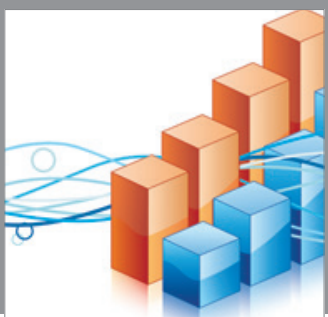

Advances in

Operations Research

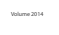

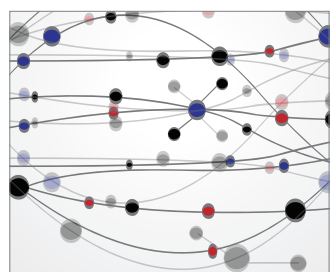

\section{The Scientific} World Journal
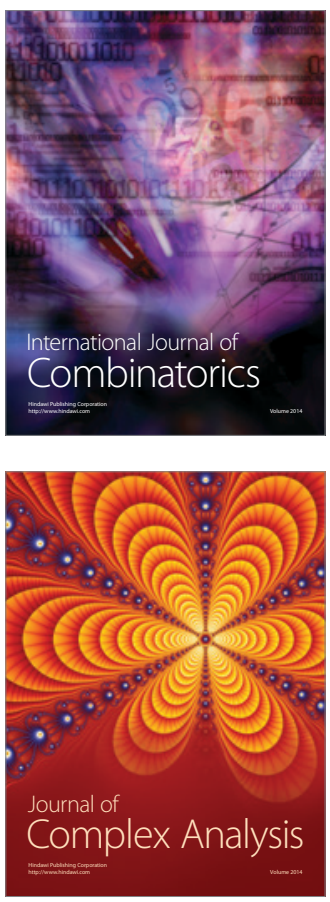

International Journal of

Mathematics and

Mathematical

Sciences
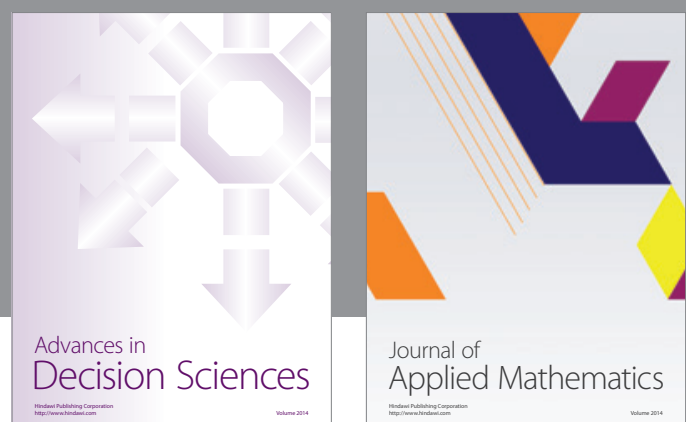

Journal of

Applied Mathematics
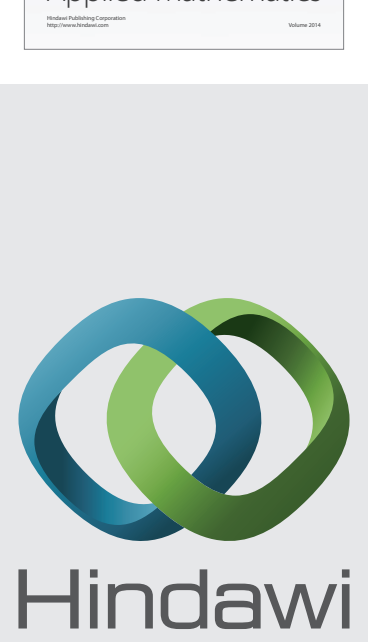

Submit your manuscripts at http://www.hindawi.com
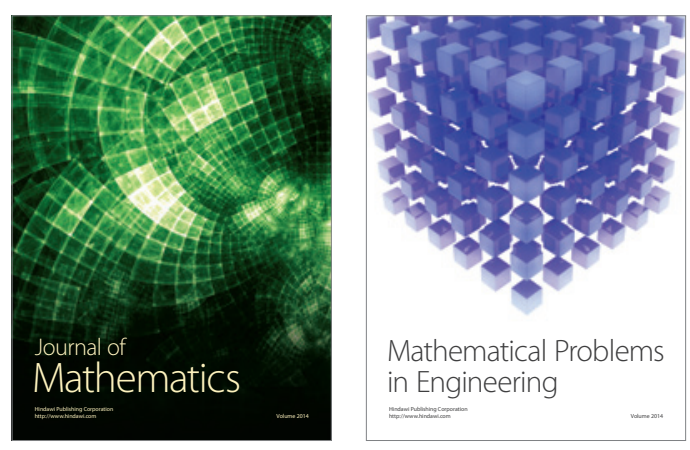

Mathematical Problems in Engineering
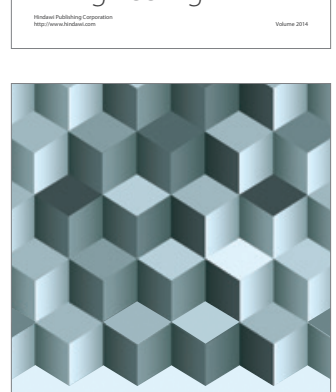

Journal of

Function Spaces
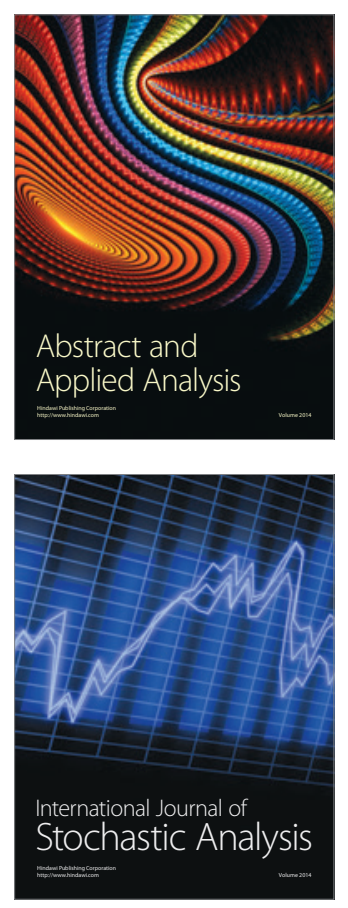

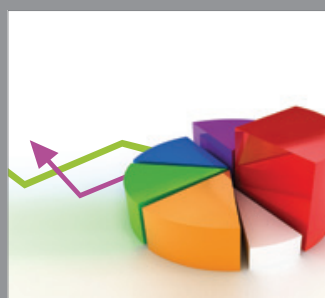

ournal of

Probability and Statistics

Promensencen
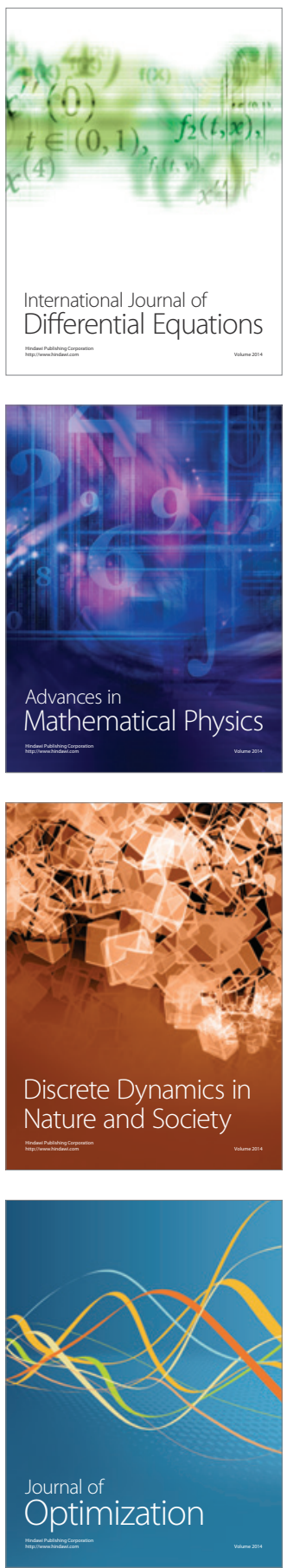